\title{
Diversity of Angiosperm Taxa in Chaar Khidirpur Area of Rajshahi, Bangladesh
}

\author{
Anjuman Ara ${ }^{1}$, Laila Al Faria ${ }^{1}$, Rony Rani ${ }^{1}$, A.H.M. Mahbubur Rahman ${ }^{2}$ \\ ${ }^{1}$ Plant Taxonomy Laboratory, Department of Botany, Faculty of Biological Sciences, University of \\ Rajshahi, Rajshahi-6205, Bangladesh \\ ${ }^{2}$ Plant Taxonomy Laboratory, Department of Botany, Faculty of Biological Sciences, University of \\ Rajshahi, Rajshahi-6205, Bangladesh
}

\begin{tabular}{|c|c|}
\hline \multirow{5}{*}{$\begin{array}{l}\text { Article Info } \\
\text { Article history: } \\
\text { Received:11 May } 2021 \\
\text { Revised: } 28 \text { July } 2021 \\
\text { Accepted:03 August } 2021\end{array}$} & Abstract \\
\hline & Purpose: The aims to investigate the angiosperm taxa in the study area. \\
\hline & The present research also documented the species diversity and important \\
\hline & Subjects and Methods: Angiosperm flora in the Chaar Khidirpur area of \\
\hline & $\begin{array}{l}\text { Rajshahi, Bangladesh was carried out from November } 2018 \text { to October } \\
2019 \text { to cover the seasonal variations. Plant parts with either flower or }\end{array}$ \\
\hline $\begin{array}{l}\text { Keywords: } \\
\text { Diversity, Angiosperms, Chaar }\end{array}$ & $\begin{array}{l}\text { fruits collected using traditional herbarium techniques to make voucher } \\
\text { specimens for documentation. }\end{array}$ \\
\hline $\begin{array}{l}\text { Khidirpur, Medicinal plants, } \\
\text { Rajshahi, Bangladesh }\end{array}$ & $\begin{array}{l}\text { Results: The result focused that a total of } 210 \text { species belonging tol } 77 \\
\text { genera under } 71 \text { families were recorded. Forty-five (45) medicinal plants } \\
\text { were used for the treatment of more than } 61 \text { diseases. }\end{array}$ \\
\hline Paper Type : & Conclusion: The present study was the first time to report angiosperm \\
\hline Research Article & rea. In this research, the status \\
\hline $\begin{array}{l}\text { Correspondence Author } \\
\text { A.H.M. Mahbubur Rahman } \\
\text { Email: drrahmanahmm @ru.ac.bd }\end{array}$ & $\begin{array}{l}\text { of occurrence has been recorded for proper conservation management and } \\
\text { sustainable utilization of the taxa resulting in } 81.33 \% \text { being common, } \\
16.74 \% \text { as rare and } 1.91 \% \text { are found as threatened in the study area. }\end{array}$ \\
\hline
\end{tabular}

\section{Introduction}

Angiosperms, approximately 300,000 species of flowering plants, the most important and maximum diverse group in the kingdom Plantae. Angiosperms represent about 80 percentages of all of the known green plants now living. The angiosperms are vascular seed flowers in which the ovule (egg) is sterilized and advanced right into a seed an enclosed hollow ovary. The ovary itself is commonly enclosed in a flower, that part of the angiospermous plant that incorporates the male or girl reproductive organs or both. Fruits are derived from the maturing floral organs of the angiospermous plant and rare therefore feature of angiosperms. The angiosperms dominate Earth's floor and vegetation in more environments, in particular terrestrial habitats than any other institution of flora. As a result, angiosperms are the maximum essential final source of meals for birds and mammals, which includes human. In addition, the flowering flowers are the most economically vital group of green plants, serving as a supply of pharmaceuticals, fibre products, timber, ornamentals and other commercial products (Ahmed et al, 2008-2009). Important research work on angiospermic plants were carried out in Bangladesh by Rahman (2013), Rahman et al. (2007), Rahman et al. (2008), Rahman et al. (2015), Rahman and Keya (2014), Kona and Rahman (2014), Sultana and Rahman (2016), Sarker and Rahman (2016), Rahman (2017) and Sarker and Rahman (2019). 
The present research was undertaken to record the diversity of angiosperms in Chaar Khidirpur area, Rajshahi, Bangladesh.

\section{Methodology and Procedures}

\section{Study area}

Chaar Khidirpur mouza is under Harian of Paba of Rajshahi district. Rajshahi is a metropolitan city, and a major urban, commercial and educational centre of Bangladesh. It is also the administrative seat of eponymous division and district. Located on the north bank of the Padma River, near the Bangladesh-India border, the city has a population of over 763,952 residents. The city is surrounded by the satellite towns of Nowhata and Katakhali, which together build an urban agglomeration of about 1 million populations. Arguably Rajshahi is the most clean and green among the cities in Bangladesh (BPC, 2001).

\section{Research methodology}

Diversity of angiospermic flora in Chaar Khidirpur area of Rajshahi, Bangladesh was carried out from November 2018 to October 2019. Plant parts with either flower of fruits collected using traditional herbarium techniques to make voucher specimens for documentation. Field identification of the collected specimens was confirmed comparing with herbarium specimens Rajshahi University Herbarium. In some cases, standard literature such as Hooker (1877), Prain (1903), and Ahmed et al. (2008-2009) were consulted for identification purpose. For update nomenclature Pasha and Uddin (2013) and Huq (1986) were also consulted. The specimens are deposited in the Herbarium, Department of Botany, Rajshahi University, Bangladesh for future reference.

\section{Plant Identification}

The collected specimens were identified by consulting different Floras and literatures. The major collected materials were identified and described up to species with the help of Hooker (1877); Prain (1903); Kirtikar and Basu (1987). For the current name and up to date nomenclature Huq (1986), Ahemd, et al. (2008-2009) and Pasha and Uddin (2013) were consulted.

\section{Results and Discussion}

Diversity of angiosperms in Chaar Khidirpur area of Rajshahi, Bangladesh conducted during November 2018 to October 2019. A total of 210 species belonging to 177 genera and 71 families were recorded. Of these, Magnoliopsida (Dicotyledones) is represented by 189 species under 158 genera and 62 families while Liliopsida (Monocotyledones) is represented by 21 species under 19 genera and 9 families. Habit analysis shows that herbs, shrubs, climbers and trees are represented by 102, 29, 29, 50 species respectively (Table 1). Asteraceae, Cucurbitaceae, Euphorbiaceae, Fabaceae, Solanaceae, Acanthaceae, Moraceae are the dominant families with high species diversity. For each species scientific name, local name, 
habit, flowering time, status of occurrence, voucher number and family were provided. Of 210 species recorded here, herbs are represented by $48.32 \%$, shrubs by $13.87 \%$, climber $13.87 \%$, Trees by $23.92 \%$ species (Table 1; Figure 1). Out of 210 species, $81.33 \%$ species was common, $16.74 \%$ species was rare and $1.91 \%$ species was threatened in the study area (Figure 2). Distribution of angiosperm species in the families shows variation. Asteraceae is the dominant family represented by 19 species, followed by Cucurbitaceae (17 species), Euphorbiaceae (11 species), Fabaceae (11species), Solanaceae (10 species), Acanthaceae (9 species), Amaranthaceae ( 7 species) and Moraceae ( 7 species). A single species is represented by 32 families while 2 to 6 species is represented by 31 families (Table 1 and 2).

Based on the study a preliminary list of angiosperm diversity in Chaar Khidirpur area of Rajshahi, Bangladesh conducted during November 2018 to October 2019. A total of 210 species belonging to 177 Genera under 71 families were recorded. (Table 1). The collected information is comparable with the result of other studies in Bangldesh. A total of 243 species belonging to 195 genera under 95 families were recorded in Khagrachhari district (Islam et.al, 2009). A total of 374 species belonging to 264 genera under 84 families were recorded in Lawachara National Park (Uddin \& Hassan, 2010). A total of 153 species belonging to 120 genera under 52 families were recorded in Runctia Sal Forest (Tutul et. al, 2010). A total of 245 species belonging to 183 genera and 72 families are documented in Habiganj district (Anefin et. al, 2011). A total of 425 species belonging to 321 genera 108 families are recorded in Rajshahi district (Rahman, 2013). A total of 302 species belonging to 243 genera 84 families are recorded in Bangladesh Police Academy, Rajshahi (Rahman et. al, 2014). No published information recorded on the diversity of angiosperms in Chaar Khidirpur area of Rajshahi, Bangladesh.

The important medicinal plants were used by the local in the study area were documented. A total of 45 medicinal plants were collected and recorded for their use in 61 diseases (Table 3). These medicinal weeds are used by the local people to cure the following diseases, especially for constipation, dysentery, diuretic, indigestion, high blood pressure, diabetes, headache, heart disease, liver disease, pain, night blindness, skin disease, burning sensation, hair disease. abdominal pain, bronchitis, asthma, stomachic, astringent, cough and cold, piles, anemia, eczema, syphilis, urinary problem, ringworm, sexual disease, leprosy, vomiting and others. Similar works were done in Bangladesh like Ghani (2003), Yusuf et al. (2009), Anisuzzaman et al.(2007), Rahman (2021a), Rahman (2021b), Rahman and Khatun (2020), Choudhury and Rahmatullah (2012), Faruque and Uddin (2014) and Uddin et al. (2008).

Table 1: Showing the families of the plant species recorded.

\begin{tabular}{|l|l|l|l|l|l|}
\hline $\begin{array}{l}\text { Sl. } \\
\text { No }\end{array}$ & Family Name & $\begin{array}{l}\text { No. of the Herb } \\
\text { species }\end{array}$ & $\begin{array}{l}\text { No. of the } \\
\text { Shurb species }\end{array}$ & $\begin{array}{l}\text { No. of the } \\
\text { Climber species }\end{array}$ & $\begin{array}{l}\text { No. of the Tree } \\
\text { species }\end{array}$ \\
\hline 1. & Acanthaceae & 7 & 1 & 1 & - \\
\hline 2. & Aloeaceae & 1 & - & - & - \\
\hline 3. & Amaranthaceae & 7 & - & - & - \\
\hline 4. & Anacardiaceae & - & - & - & 2 \\
\hline 5. & Annonaceae & - & - & - & 3 \\
\hline 6. & Apiaceae & 2 & - & - & - \\
\hline 7. & Apocynaceae & 3 & - & - & 2 \\
\hline
\end{tabular}




\begin{tabular}{|c|c|c|c|c|c|}
\hline 8. & Araceae & 4 & - & - & - \\
\hline 9. & Arecaceae & - & - & - & 4 \\
\hline 10. & Asclepiadaceae & - & 2 & - & - \\
\hline 11. & Asteraceae & 19 & - & 1 & - \\
\hline 12. & Basellaceae & - & - & 1 & - \\
\hline 13. & Bombacaceae & - & - & - & 1 \\
\hline 14. & Boraginaceae & 1 & - & - & - \\
\hline 15. & Brassicaceae & 3 & - & - & - \\
\hline 16. & Caesalpiniaceae & - & 2 & - & 2 \\
\hline 17. & Cannaceae & 1 & - & - & - \\
\hline 18. & Capparaceae & 1 & - & - & - \\
\hline 19. & Caricaceae & - & - & - & 1 \\
\hline 20. & Chenopodiaceae & 3 & - & - & - \\
\hline 21. & Combretaceae & - & - & - & 1 \\
\hline 22. & Commelinaceae & 1 & - & - & - \\
\hline 23. & Convolvulaceae & 1 & 1 & 2 & - \\
\hline 24. & Cosiaceae & 1 & - & - & - \\
\hline 25. & Crassulaceae & 1 & - & - & - \\
\hline 26. & Cucurbitaceae & - & - & 17 & - \\
\hline 27. & Cyperaceae & 2 & - & - & - \\
\hline 28. & Dilleniaceae & - & - & - & 1 \\
\hline 29. & Dioscoriaceae & - & - & 1 & - \\
\hline 30. & Elaeocarpaceae & - & - & - & 1 \\
\hline 31. & Euphorbiaceae & 4 & 5 & - & 2 \\
\hline 32. & Fabaceae & 7 & 1 & 1 & 2 \\
\hline 33. & Gentianaceae & 1 & - & - & - \\
\hline 34. & Lamiaceae & 3 & - & - & - \\
\hline 35. & Lauraceae & - & - & - & 1 \\
\hline 36. & Lemnaceae & 1 & - & - & - \\
\hline 37. & Liliaceae & 2 & - & 1 & - \\
\hline 38. & Lythraceae & - & 1 & - & 1 \\
\hline 39. & Malvaceae & 3 & 1 & - & - \\
\hline 40. & Meliaceae & - & - & - & 2 \\
\hline 41. & Menispermaceae & - & - & 2 & - \\
\hline 42. & Mimosaceae & - & - & - & 3 \\
\hline 43. & Molluginaceae & 1 & - & - & - \\
\hline 44. & Moraceae & - & - & - & 7 \\
\hline 45. & Moringaceae & - & - & - & 1 \\
\hline 46. & Musaceae & 1 & - & - & - \\
\hline 47. & Myrtaceae & - & - & - & 3 \\
\hline 48. & Oxalidaceae & 1 & - & - & 1 \\
\hline 49. & Orobanchaceae & 1 & - & - & - \\
\hline 50. & Papaveraceae & 1 & - & - & - \\
\hline 51. & Pedaliaceae & 1 & - & - & - \\
\hline 52. & Piperaceae & 1 & - & - & - \\
\hline 53. & Poaceae & 1 & 2 & - & - \\
\hline 54. & Polygonaceae & 1 & - & - & - \\
\hline 55. & Portulaceae & 1 & - & - & - \\
\hline 56. & Punieaceae & - & 1 & - & - \\
\hline 57. & Rhamnaceae & - & - & - & 1 \\
\hline 58. & Rosaceae & - & 2 & - & - \\
\hline 59. & Rubiaceae & - & 1 & - & 1 \\
\hline 60. & Rutaceae & - & 2 & - & 2 \\
\hline
\end{tabular}




\begin{tabular}{|l|l|l|l|l|l|}
\hline 61. & Sapindaceae & - & - & - & 1 \\
\hline 62. & Sapotaceae & - & - & - & 2 \\
\hline 63. & Scrophulariaceae & 1 & - & - & - \\
\hline 64. & Smilaceae & - & - & 1 & - \\
\hline 65. & Solanaceae & 8 & 2 & - & - \\
\hline 66. & Sterculiaceae & - & 1 & - & - \\
\hline 67. & Ulmaceae & - & - & - & 1 \\
\hline 68. & Urticaceae & 1 & - & - & - \\
\hline 69. & Verbenaceae & 1 & 5 & - & - \\
\hline 70. & Vitaceae & - & - & 1 & - \\
\hline 71. & Zingiberaceae & 2 & - & - & - \\
\hline
\end{tabular}

Table 2. Documentation of Angiosperm Taxa in Chaar Khidirpur, Rajshahi, Bangladesh

\begin{tabular}{|c|c|c|c|c|c|c|}
\hline Scientific name & $\begin{array}{c}\text { Bangla } \\
\text { name }\end{array}$ & Family name & Habit & $\begin{array}{c}\text { Status of } \\
\text { occurrence }\end{array}$ & $\begin{array}{c}\text { Flowering } \\
\text { time }\end{array}$ & Voucher no. \\
\hline $\begin{array}{l}\text { Andrographis paniculata } \\
\text { (Burm. f.) Wall ex Ness. }\end{array}$ & \begin{tabular}{|l|} 
Kalameg \\
$\mathrm{h}$
\end{tabular} & Acanthaceae & Herb & $\mathrm{C}$ & Jan-Mar & AA 108 \\
\hline Barleria prionitis $\mathrm{L}$. & $\begin{array}{l}\text { Kania- } \\
\text { janti }\end{array}$ & Acanthaceae & Herb & $\mathrm{C}$ & Nov-Feb & AA 178 \\
\hline $\begin{array}{l}\text { Hemigraphis hirta (Valil) } \\
\text { T.Anders. }\end{array}$ & Buriana & Acanthaceae & Herb & $\mathrm{C}$ & Jan-Jul & AA 174 \\
\hline $\begin{array}{l}\text { Hygrophila schulli (Buch- } \\
\text { Ham.) M.R. \& S.N.Almeida. }\end{array}$ & $\begin{array}{l}\text { Kule } \\
\text { kharha }\end{array}$ & Acanthaceae & Herb & $\mathrm{R}$ & Oct-Jan & AA 109 \\
\hline Justicia gendarussa Burm. f. & $\begin{array}{l}\text { Jagath } \\
\text { madan }\end{array}$ & Acanthaceae & Herb & $\mathrm{C}$ & Apr-Aug & AA 201 \\
\hline Justicia adhatoda L. & Basok & Acanthaceae & Shrub & $\mathrm{R}$ & Jan-Apr & AA 179 \\
\hline $\begin{array}{l}\text { Nelsonia canescens (Lamk.) } \\
\text { Spreng. }\end{array}$ & Paramul & Acanthaceae & Herb & $\mathrm{C}$ & Oct-Feb & AA 110 \\
\hline Ruellia tuberosa $\mathrm{L}$. & Chatpoty & Acanthaceae & Herb & $\mathrm{C}$ & Jan-Dec & AA 180 \\
\hline $\begin{array}{ll}\text { Thunbergia } & \text { grandiflora } \\
\text { (Roxh. ex Rotller) Roth. }\end{array}$ & Nillata & Acanthaceae & Clim & $\mathrm{R}$ & Jan-Dec & AA 187 \\
\hline Aloe vera $(\mathrm{L}$.$) Bum \mathrm{f}$. & $\begin{array}{l}\text { Ghrita } \\
\text { kumari }\end{array}$ & Aloeaceae & Herb & $\mathrm{C}$ & Sep-Dec & AA129 \\
\hline Amaranthus spinosus $\mathrm{L}$. & $\begin{array}{l}\text { Katanote } \\
\mathrm{y}\end{array}$ & Amaranthaceae & Herb & $\mathrm{C}$ & Jan-Dec & AA 205 \\
\hline Amaranthus viridis $\mathrm{L}$. & Notyshak & Amaranthaceae & Herb & $\mathrm{C}$ & Jan-Dec & AA 211 \\
\hline Achyranthes aspera $\mathrm{L}$. & Aparg & Amaranthaceae & Herb & $\mathrm{C}$ & Jan-Dec & AA 210 \\
\hline $\begin{array}{l}\text { Aerva lanata (L.) .juss. ex } \\
\text { Schult. }\end{array}$ & Chaya & Amaranthaceae & Herb & $\mathrm{C}$ & Apr-Jul & AA 134 \\
\hline Alternanthera sessilis $\mathrm{R} . \mathrm{Br}$ & Chanshi & Amaranthaceae & Herb & $\mathrm{C}$ & Jan-Dec & AA 139 \\
\hline Celosia cristata $\mathrm{L}$. & Morogful & Amaranthaceae & Herb & $\mathrm{C}$ & Jan-Dec & AA 135 \\
\hline Dirgeria muricata (L.) Mart. & \begin{tabular}{|l|} 
Gungatik \\
a \\
\end{tabular} & Amaranthaceae & Herb & $\mathrm{T}$ & Feb-Jun & AA 147 \\
\hline Magnifera indica $\mathrm{L}$. & Aam & Anacardiaceae & Tree & $\mathrm{C}$ & Jan-Apr & AA 162 \\
\hline Spondias pinnata (L.f.) Kurz. & Aamra & Anacardiaceae & Tree & $\mathrm{C}$ & Feb-Jun & AA 131 \\
\hline Annona squamosa $\mathrm{L}$. & Sarifa & Annonaceae & Tree & $\mathrm{C}$ & Mar-Jul & AA 37 \\
\hline Annona reticulata $\mathrm{L}$. & $\begin{array}{l}\text { Nona, } \\
\text { Ata } \\
\end{array}$ & Annonaceae & Tree & $\mathrm{C}$ & Oct-Jan & AA 29 \\
\hline $\begin{array}{l}\text { Polyalthia longifolia (Sonn.) } \\
\text { Thw. }\end{array}$ & Debdaru & Annonaceae & Tree & $\mathrm{C}$ & Mar-Oct & AA 21 \\
\hline Centella asiatica (L.) Urban. & Thankuni & Apiaceae & Herb & $\mathrm{C}$ & Jan-Dec & AA142 \\
\hline Daucus carota $\mathrm{L}$. & Gajor & Apiaceae & Herb & $\mathrm{C}$ & May-Aug & AA165 \\
\hline Alstonia scholaris (L.) R. Br. & Chatim & Apocynaceae & Tree & $\mathrm{R}$ & Nov-May & AA 96 \\
\hline Carissa carandas $\mathrm{L}$. & $\begin{array}{l}\text { Karamch } \\
\text { a }\end{array}$ & Apocynaceae & Herb & $\mathrm{C}$ & Jan-Dec & AA 166 \\
\hline
\end{tabular}




\begin{tabular}{|c|c|c|c|c|c|c|}
\hline $\begin{array}{l}\text { Catharanthus roseus (L.) G. } \\
\text { Don. }\end{array}$ & $\begin{array}{l}\text { Nayantar } \\
\text { a } \\
\end{array}$ & Apocynaceae & Herb & $\mathrm{C}$ & Jan-Dec & AA 136 \\
\hline $\begin{array}{ll}\text { Rauvolfia serpentina } & \text { (L.) } \\
\text { Benth ex Kurz. }\end{array}$ & $\begin{array}{l}\text { Sarpa } \\
\text { gandha }\end{array}$ & Apocynaceae & Herb & $\mathrm{R}$ & Jan-Dec & AA 204 \\
\hline Thevetia peruviana Pers. & $\begin{array}{l}\text { Halide } \\
\text { karabi }\end{array}$ & Apocynaceae & Tree & $\mathrm{C}$ & Jan-Dec & AA 98 \\
\hline $\begin{array}{l}\text { Alocasia macrorrhizos (L.) G. } \\
\text { Don. }\end{array}$ & $\begin{array}{l}\text { Mankocb } \\
\mathrm{u}\end{array}$ & Araceae & Herb & $\mathrm{C}$ & Jul-Oct & AA190 \\
\hline $\begin{array}{l}\text { Amorphophallus } \\
\text { campanulalus } \\
\text { Bl.ex.Dense. }\end{array}$ & Olkochu & Araceae & Herb & $\mathrm{C}$ & May-Nov & AA121 \\
\hline $\begin{array}{l}\text { Colocasia } \\
\text { Schott. }\end{array}$ & Kochu & Araceae & Herb & $\mathrm{C}$ & May-Oct & AA191 \\
\hline $\begin{array}{lll}\begin{array}{l}\text { Typhonium } \\
\text { Schott. }\end{array} & & \\
\end{array}$ & Camgash & Araceae & Herb & $\mathrm{C}$ & Apr-Oct & AA206 \\
\hline Areca catechu $\mathrm{L}$. & Shupari & Arecaceae & Tree & $\mathrm{C}$ & Jan-Dec & AA119 \\
\hline Borassus flabellifer $\mathrm{L}$. & Taal & Arecaceae & Tree & $\mathrm{C}$ & Jan-Oct & AA189 \\
\hline Cocos nucifera $\mathrm{L}$. & Narkel & Arecaceae & Tree & $\mathrm{C}$ & Jan-Dec & AA158 \\
\hline Phoenix sylvextrix Roxb. & Khejur & Arecaceae & Tree & $\mathrm{C}$ & Dec-May & AA120 \\
\hline $\begin{array}{l}\text { Calotropis procera (Ait.) R. } \\
\text { Br. }\end{array}$ & Akondo & Asclepiadaccae & Shrub & $\mathrm{C}$ & Apr-May & AA 137 \\
\hline Calotropis gigantea $(\mathrm{L}$.$) R.Br.$ & Akondo & Asclepiadaceae & Shrub & $\mathrm{C}$ & Apr-May & AA 168 \\
\hline Ageratum conyzoides $\mathrm{L}$. & Ochunti & Asteraceae & Herb & $\mathrm{C}$ & Nov-Jun & AA112 \\
\hline Blumea lacera (Burm. f.) DC. & $\begin{array}{l}\text { Boro } \\
\text { kucksim }\end{array}$ & Asteraceae & Herb & $\mathrm{C}$ & Nov-Jul & AA182 \\
\hline $\begin{array}{l}\text { Chromolaena odorata } \mathrm{L} . \\
\text { King. \& Robinson. }\end{array}$ & $\begin{array}{l}\text { German } \\
\text { lata }\end{array}$ & Asteraceae & Herb & $\mathrm{R}$ & Nov-May & AA130 \\
\hline Cirsium arvense (L.) Scop. & $\begin{array}{l}\text { Shial } \\
\text { Kanta } \\
\end{array}$ & Asteraceae & Herb & $\mathrm{C}$ & Feb-Jun & AA113 \\
\hline Eclipta alba (L.) Hassk. & $\begin{array}{l}\text { Kalokesh } \\
\text { i }\end{array}$ & Asteraceae & Herb & $\mathrm{R}$ & Jan-Dec & AA152 \\
\hline Enhydra fluctuans Lour. & Helencha & Asteraceae & Herb & $\mathrm{C}$ & Jan-Apr & AA114 \\
\hline Gnaphalium luteo-ulbum $\mathrm{L}$. & $\begin{array}{l}\text { Bara } \\
\text { Kamra } \\
\end{array}$ & Asteraceae & Herb & $\mathrm{C}$ & Mar-Aug & AA184 \\
\hline $\begin{array}{l}\text { Grangea maderaspatana (L.) } \\
\text { Poir. }\end{array}$ & Namuti & Asteraceae & Herb & $\mathrm{R}$ & Dec-May & AA153 \\
\hline Hellianthus annuиs L. & $\begin{array}{l}\text { Surjo } \\
\text { mukhi }\end{array}$ & Asteraceae & Herb & $\mathrm{C}$ & Jan-Dec & AA115 \\
\hline Launaea aspleniifolia DC. & $\begin{array}{l}\text { Tik- } \\
\text { chana }\end{array}$ & Asteraceae & Herb & $\mathrm{C}$ & Jan-Aug & AA154 \\
\hline $\begin{array}{l}\text { Mikania cordata (Bunn f.) } \\
\text { Robinson. }\end{array}$ & $\begin{array}{ll}\text { As am } \\
\text { lata }\end{array}$ & Asteraceae & Clim & $\mathrm{C}$ & Oct-Feb & AA116 \\
\hline Sonchus asper (L.) Hill. & $\begin{array}{l}\text { Sonpalon } \\
\mathrm{g}\end{array}$ & Asteraceae & Herb & $\mathrm{C}$ & Sep-Jun & AA186 \\
\hline Spilanthes acmella (L.)L. & $\begin{array}{l}\text { Marha } \\
\text { titiga }\end{array}$ & Asteraceae & Herb & $\mathrm{R}$ & Jan-Dec & AA 155 \\
\hline $\begin{array}{lll}\text { Synedrella } & \text { nodiflora } & \text { (L.) } \\
\text { Gaertn. } & & \\
\end{array}$ & Relanodi & Asteraceae & Herb & $\mathrm{R}$ & Jan-Dec & AA 203 \\
\hline Tagetes erecta $\mathrm{L}$. & Genda & Asteraceae & Herb & $\mathrm{C}$ & Jan-Dec & AA 117 \\
\hline Tridax procumbens L. & Tridbara & Asteraceae & Herb & $\mathrm{C}$ & Jan-Dec & AA 25 \\
\hline Vernoma cinerea (L.) Less. & Kuksim & Asteraceae & Herb & $\mathrm{C}$ & Jan-Dec & AA105 \\
\hline $\begin{array}{l}\text { Wedelia trilobata (L.) A S. } \\
\text { Hitchc. }\end{array}$ & Keshraj & Asteraceae & Herb & $\mathrm{C}$ & Jan-Dec & AA 31 \\
\hline $\begin{array}{l}\text { Xanthium indicum Koen ex } \\
\text { Roxb. }\end{array}$ & Ghagra & Asteraceae & Herb & $\mathrm{C}$ & Jan-Dec & AA 157 \\
\hline Youngia japonica (L.) DC. & $\begin{array}{l}\text { Young } \\
\text { ful }\end{array}$ & Asteraceae & Herb & $\mathrm{R}$ & Aug-Jan & AA 44 \\
\hline Basella rubra L. & Pushak & Basellaceae & Clim & $\mathrm{C}$ & Nov-Feb & AA 58 \\
\hline Bombax ceiba L. & Shimul & Bombacaceae & Tree & $\mathrm{C}$ & Feb-Apr & AA 82 \\
\hline
\end{tabular}

Copyright @ 2020-2021, Journal of Scientific Research in Medical and Biological Sciences (JSRMBS), Under the license CC BY- 4.0 


\begin{tabular}{|c|c|c|c|c|c|c|}
\hline Heliotropium indicum $\mathrm{L}$. & Hatishur & Boraginaceae & Herb & $\mathrm{C}$ & Jan-Dec & AA 123 \\
\hline $\begin{array}{l}\text { Brassica oleracea } \text { L. var. } \\
\text { botrytis L. }\end{array}$ & Phulkopi & Brassicaceae & Herb & $\mathrm{C}$ & Nov-Apr & AA 126 \\
\hline $\begin{array}{l}\begin{array}{l}\text { Brassica oleracea L. var } \\
\text { capitata L. }\end{array} \\
\end{array}$ & $\begin{array}{l}\text { Badha } \\
\text { kopi }\end{array}$ & Brassicaceae & Herb & $\mathrm{C}$ & Nov-Apr & AA 144 \\
\hline Raphanus sativus $\mathrm{L}$. & Mula & Brassicaceae & Herb & $\mathrm{C}$ & Jan-May & AA 170 \\
\hline Delonix regia (Boyer) Raf. & $\begin{array}{l}\text { Krishno } \\
\text { chura }\end{array}$ & Caesalpiniaceae & Tree & $\mathrm{C}$ & Apr-Sep & AA 194 \\
\hline Senna sophera (L.) Roxb. & $\begin{array}{l}\text { Kalka } \\
\text { shunda }\end{array}$ & Caesalpiniaceae & Shrub & $\mathrm{C}$ & Dec-Mar & AA 198 \\
\hline Senna alata (L.) Roxb. & $\begin{array}{l}\text { Dad } \\
\text { mardan }\end{array}$ & Caesalpiniaceae & Shrub & $\mathrm{R}$ & Sep-Jan & AA 100 \\
\hline Tamarimdus indica $\mathrm{L}$. & Tentul & Caesalpiniaceae & Tree & $\mathrm{C}$ & Apr-Dec & AA 68 \\
\hline Canna indica $\mathrm{L}$. & Kolaboti & Cannaceae & Herb & $\mathrm{C}$ & Apr-Nov & AA 71 \\
\hline Cleome viscosa $\mathrm{L}$. & \begin{tabular}{|l|} 
Hurhuria \\
\end{tabular} & Capparaceae & Herb & $\mathrm{R}$ & Jan-Dec & AA 76 \\
\hline Carica papaya $\mathrm{L}$. & Pape & Caricaceae & Tree & $\mathrm{C}$ & Jan-Dec & AA 09 \\
\hline Chenopodium album $\mathrm{L}$. & Bothua & Chenopodiaceae & Herb & $\mathrm{C}$ & Dec-Mar & AA 17 \\
\hline $\begin{array}{l}\text { Chenopodium ambrosioides } \\
\text { L. }\end{array}$ & Banbotua & Chenopodiaceae & Herb & $\mathrm{C}$ & Jan-Dec & AA 52 \\
\hline Spinacia oleracea $\mathrm{L}$. & $\begin{array}{l}\text { Palong } \\
\text { shak }\end{array}$ & Chenopodiaecae & Herb & $\mathrm{C}$ & Feb-Mar & AA 88 \\
\hline $\begin{array}{l}\text { Terminalia arjuna (Roxb. ex. } \\
\text { DC) }\end{array}$ & Arjun & Combretaceae & Tree & $\mathrm{C}$ & Apr-Jul & AA 93 \\
\hline Commelina benghalensis L. & Kanshira & Commelinaceae & Herb & $\mathrm{C}$ & Apr-Nov & AA 40 \\
\hline Evolvulus nummularius (L.) L. & Bhuiokra & Convolvulaceae & Herb & $\mathrm{C}$ & Jan-Dec & AA 73 \\
\hline Ipomoea batatas (L.) Lamk. & Mistialu & Convolvulaceae & Clim & $\mathrm{C}$ & Jan-Dec & AA 48 \\
\hline $\begin{array}{l}\text { Ipomoea fistulosa Mart. ex. } \\
\text { Choisy in DC. }\end{array}$ & $\begin{array}{l}\text { Dholkaln } \\
\text { ii } \\
\end{array}$ & Convolvulaceae & Shrub & $\mathrm{C}$ & Jan-Dec & AA 55 \\
\hline Ipomoea aquatica Forssk. & $\begin{array}{l}\text { Kalmisha } \\
\mathrm{k}\end{array}$ & Convolvulaceae & Clim & $\mathrm{C}$ & Jan-Oct & AA 60 \\
\hline $\begin{array}{l}\text { Costus speciosus (Koenig ex } \\
\text { Retz) Smith. }\end{array}$ & Keumul & Costaceae & Herb & $\mathrm{R}$ & Sep-Dec & AA 65 \\
\hline Kalanchoe laciniala (L.) Pers. & \begin{tabular}{|l} 
Himsagor \\
\end{tabular} & Crassulaceae & Herb & $\mathrm{R}$ & Jan-Mar & AA 124 \\
\hline $\begin{array}{l}\text { Benincasa hispida (Thurb.) } \\
\text { Cogn. }\end{array}$ & $\begin{array}{l}\text { Chal } \\
\text { kumra }\end{array}$ & Cucurbitaceae & Clim & $\mathrm{C}$ & May-Nov & AA 75 \\
\hline Cucunus sativus $\mathrm{L}$. & Sosha & Cucurbitaceae & Clim & $\mathrm{C}$ & Apr-Sep & AA 103 \\
\hline $\begin{array}{l}\text { Citrullus lanatus (Thunb.) } \\
\text { Mat. \& Nak. }\end{array}$ & Tormuj & Cucurbitaceae & Clim & $\mathrm{C}$ & Mar-Sep & AA 35 \\
\hline Coccinia grandis (L.) Voigt. & $\begin{array}{l}\text { Telakuch } \\
\text { a }\end{array}$ & Cucurbitaceae & Clim & $\mathrm{C}$ & Mar-Dec & AA 50 \\
\hline Cucunus melo L. & Bangi & Cucurbitaceae & Clim & $\mathrm{C}$ & Mar-Jul & AA 58 \\
\hline Cucurbita maxima Duch. & $\begin{array}{l}\text { Misti } \\
\text { kumra }\end{array}$ & Cucurbitaceae & Clim & $\mathrm{C}$ & Mar-Aug & AA 91 \\
\hline $\begin{array}{l}\text { Gymnopetalum } \\
\text { cochinchinense (Lour.) Kurz. }\end{array}$ & $\begin{array}{l}\text { Bati } \\
\text { Jhinga }\end{array}$ & Cucurbitaceae & Clim & $\mathrm{R}$ & Jul-Dec & AA 150 \\
\hline $\begin{array}{l}\text { Lagenaria siceraria (Monila) } \\
\text { Standl. }\end{array}$ & Panilau & Cucurbitaceae & Clim & $\mathrm{C}$ & Feb-May & AA 172 \\
\hline Luffa acutangula (L.) Roxb. & Jhinga & Cucurbitaceae & Clim & $\mathrm{C}$ & Apr-Oct & AA 176 \\
\hline Luffa cynlidrica (L.) Roem. & Dhundul & Cucurbitaceae & Clim & $\mathrm{C}$ & Jun-Dec & AA 185 \\
\hline $\begin{array}{l}\text { Momordica cochinchinensis } \\
\text { (Lour.) Spreng. }\end{array}$ & Kakrol & Cucurbitaceae & Clim & $\mathrm{C}$ & Jul-Nov & AA 193 \\
\hline Momordica charantia $\mathrm{L}$. & Korolla & Cucurbitaceae & Clim & $\mathrm{C}$ & May-Oct & AA 05 \\
\hline $\begin{array}{l}\text { Mukia maderaspatana (L.) M. } \\
\text { Roem. }\end{array}$ & Agmuki & Cucurbitaceae & Clim & $\mathrm{R}$ & Jun-Dec & AA 23 \\
\hline Trichossasthes cucumerina L. & $\begin{array}{l}\text { Ban } \\
\text { chichinga }\end{array}$ & Cucurbitaceae & Clim & $\mathrm{C}$ & Apr-Jun & AA 181 \\
\hline Trichossasthes dioica Roxb. & Potol & Cucurbitaceae & Clim & $\mathrm{C}$ & Apr-Sep & AA 195 \\
\hline Trichossasthes tricuspidata & Makal & Cucurbitaceae & Clim & $\mathrm{R}$ & Jul-Dec & AA 140 \\
\hline
\end{tabular}




\begin{tabular}{|c|c|c|c|c|c|c|}
\hline Lour. & & & & & & \\
\hline Trichossasthes anguina $\mathrm{L}$. & $\begin{array}{l}\text { Chiching } \\
\text { a }\end{array}$ & Cucurbitaceae & Clim & $\mathrm{C}$ & Apr-Jun & AA 192 \\
\hline Cyperus rotundus $\mathrm{L}$. & Muthagas & Cyperaceae & Herb & $\mathrm{C}$ & Sep-Feb & AA 183 \\
\hline $\begin{array}{l}\text { Kyllinga nemoralis (J.R. Forst. } \\
\& \text { G. Forst.) Dandv ex } \\
\text { Hutchins. \& Dal. }\end{array}$ & Nirbishi & Cyperaceae & Herb & $\mathrm{C}$ & Jun-Sep & AA 148 \\
\hline Dillenia indica $\mathrm{L}$. & Chalta & Dilleniaceae & Tree & $\mathrm{R}$ & May-Oct & AA 171 \\
\hline Dioscorea alata $\mathrm{L}$. & $\begin{array}{l}\text { Chupri } \\
\text { alu }\end{array}$ & Dioscoriaceae & Clim & $\mathrm{R}$ & Oct-Dec & AA207 \\
\hline $\begin{array}{l}\text { Elaeocarpus tectorius (Lour.) } \\
\text { Poir. }\end{array}$ & Jolpai & Elaeocarpaceae & Tree & $\mathrm{R}$ & May-Oct & AA 18 \\
\hline Acalypha indica $L$. & $\begin{array}{l}\text { Mukta } \\
\text { jhuri }\end{array}$ & Euphorbiaceae & Herb & $\mathrm{C}$ & Dec-Apr & AA 26 \\
\hline $\begin{array}{l}\text { Croton bonplandianus } \\
\text { Baill. }\end{array}$ & $\begin{array}{l}\text { Ban } \\
\text { morich }\end{array}$ & Euphorbiaceae & Herb & $\mathrm{C}$ & Jan-Dec & AA 188 \\
\hline Euphorbia hirta L. & Dudhiya & Euphorbiaceae & Herb & $\mathrm{C}$ & Jan-Dec & AA 200 \\
\hline Jatropha gossypifolia $\mathrm{L}$. & $\begin{array}{l}\text { Lal } \\
\text { bherenda }\end{array}$ & Euphorbiaceae & Shrub & $\mathrm{C}$ & Apr-Aug & AA 143 \\
\hline Jatropha curcas $\mathrm{L}$. & $\begin{array}{l}\text { Jamalgot } \\
\text { a }\end{array}$ & Euphorbiaceae & Shrub & $\mathrm{R}$ & Sep-Dec & AA 151 \\
\hline Manihot esculenta Crantz. & Kasava & Euphorbiaceae & Shrub & $\mathrm{C}$ & Sep-Jan & AA 202 \\
\hline Phyllanthus niruri L. & Bhuiamla & Euphorbiaceae & Herb & $\mathrm{C}$ & Aug-Oct & AA 175 \\
\hline Phyllanthus reticulatus Poir. & Chitki & Euphorbiaceae & Shrub & $\mathrm{C}$ & Mar-Oct & AA 42 \\
\hline Phyllanthus emblica L. & Amloki & Euphorbiaceae & Tree & $\mathrm{R}$ & Mar-Sep & AA 59 \\
\hline Ricinus communis $\mathrm{L}$. & Bherenda & Euphorbiaceae & Shrub & $\mathrm{C}$ & Jan-Dec & AA 208 \\
\hline Trewia nodiflora $\mathrm{L}$. & $\begin{array}{l}\text { Batul, } \\
\text { latim }\end{array}$ & Euphorbiaceae & Tree & $\mathrm{C}$ & Feb-Aug & AA 177 \\
\hline Arachis hypogea $\mathrm{L}$. & $\begin{array}{l}\text { China } \\
\text { badam }\end{array}$ & Fabaceae & Herb & $\mathrm{C}$ & Mar-Dec & AA 209 \\
\hline Cajanus cajan (L.) Millsp. & Arhar & Fabaceae & Shrub & $\mathrm{C}$ & Dec-Apr & AA 111 \\
\hline Desmodium triflorum (L.) DC & Kudalia & Fabaceae & Herb & $\mathrm{C}$ & Jan-Dec & AA 07 \\
\hline Dalbergia sissoo Roxb. & $\begin{array}{l}\text { Sissoo } \\
\text { Gachh }\end{array}$ & Fabaceae & Tree & $\mathrm{C}$ & Mar-Jun & AA 32 \\
\hline $\begin{array}{l}\text { Desmodium gangeticum (L.) } \\
\text { DC. }\end{array}$ & Salpani & Fabaceae & Herb & $\mathrm{C}$ & Apr-Nov & AA 97 \\
\hline Erythrina variegata $\mathrm{L}$. & Madar & Fabaceae & Tree & $\mathrm{C}$ & Feb-Mar & AA 156 \\
\hline Lablab purpureus (L.) Sweet. & Shim & Fabaceae & Clim & $\mathrm{C}$ & Nov-Mar & AA 199 \\
\hline Lathyrus sativus $\mathrm{L}$. & Khesari & Fabaceae & Herb & $\mathrm{C}$ & Feb-Sep & AA 79 \\
\hline Lens culinaris Medic. & Masur & Fabaceae & Herb & $\mathrm{C}$ & Dec-Mar & AA 161 \\
\hline Melilotus alba Desr. & $\begin{array}{l}\text { Sada } \\
\text { Methi }\end{array}$ & Fabaceae & Herb & $\mathrm{R}$ & Mar-Oct & AA 196 \\
\hline Vicia sativa $\mathrm{L}$. & Ankari & Fabaceae & Herb & $\mathrm{C}$ & Jul-Nov & AA 167 \\
\hline Exacum pedunculatum $\mathrm{L}$. & $\begin{array}{l}\text { Chiratta } \\
\mathrm{m}\end{array}$ & Gentianaceae & Herb & $\mathrm{R}$ & Feb-Apr & AA 133 \\
\hline Sesamum indicum L. & Til & Pedaliaceae & Herb & $\mathrm{C}$ & Feb-Oct & AA 159 \\
\hline Leonurus sibiricus L. & $\begin{array}{l}\text { Roklodro } \\
\mathrm{n}\end{array}$ & Lamiaceae & Herb & $\mathrm{C}$ & Jan-Dec & AA 197 \\
\hline Leucas aspera (Willd) Link. & \begin{tabular}{|l|} 
Shetodro \\
$\mathrm{n}$
\end{tabular} & Lamiaceae & Herb & $\mathrm{C}$ & Jan-Dec & AA 77 \\
\hline Ocimum tenuiflorum $\mathrm{L}$. & Tulsi & Lamiaceae & Herb & $\mathrm{R}$ & Jan-Dec & AA 47 \\
\hline Litsea glutinosa (Lour.) Rob. & Menda & Lauraceae & Tree & $\mathrm{T}$ & Apr-Jan & AA 141 \\
\hline Lemna perpusilla Torrey & $\begin{array}{l}\text { Khudi } \\
\text { pana }\end{array}$ & Lemnaceae & Herb & C & Jan-Dec & AA 169 \\
\hline Allium sativum $\mathrm{L}$. & Rosun & Liliaceae & Herb & $\mathrm{C}$ & Feb-Apr & AA 84 \\
\hline Allium cepa $\mathrm{L}$. & Piyaj & Liliaceae & Herb & $\mathrm{C}$ & Feb-Jun & AA 15 \\
\hline Asparagus racemosus Willd. & Satamili & Liliaceae & Clim & $\mathrm{R}$ & Nov-Mar & AA 54 \\
\hline $\begin{array}{l}\text { Lagestroemia speciosa (L.) } \\
\text { Pers. }\end{array}$ & Jarul & Lythraceae & Tree & $\mathrm{C}$ & Apr-Aug & AA 30 \\
\hline
\end{tabular}




\begin{tabular}{|c|c|c|c|c|c|c|}
\hline Lawsonia inermis $\mathrm{L}$. & Mehedi & Lythraceae & Shrub & $\mathrm{C}$ & Jun-Dec & AA 56 \\
\hline $\begin{array}{l}\text { Abelmoschus esculentus (L.) } \\
\text { Moench. }\end{array}$ & Bhindi & Malvaceae & Herb & $\mathrm{C}$ & Jan-Dec & AA 160 \\
\hline Abutilon indicum (L.) Sweet. & Petari & Malvaceae & Herb & $\mathrm{C}$ & Jul-Apr & AA 95 \\
\hline Hibiscus rosa-sinensis L. & Joba & Malvaceae & Shrub & $\mathrm{C}$ & Jan-Dec & AA 92 \\
\hline Sida cordifolia $\mathrm{L}$. & Berela & Malvaceae & Herb & $\mathrm{C}$ & Sep-Dec & AA 99 \\
\hline Azadirachta indica A. Juss. & Neem & Meliaceae & Tree & $\mathrm{C}$ & Mar-Jul & AA 33 \\
\hline Swietenia mahagoni Jacq. & $\begin{array}{l}\text { Mahagon } \\
\mathrm{i}\end{array}$ & Meliaceae & Tree & $\mathrm{C}$ & Apr-Nov & AA 38 \\
\hline $\begin{array}{l}\text { Stephania japonica (Thunb.) } \\
\text { Miers. }\end{array}$ & Akanadi & Menispermaceae & Clim & $\mathrm{R}$ & Jan-Dec & AA 41 \\
\hline $\begin{array}{l}\text { Tinospora cordifolia (Willd.) } \\
\text { Hook. f \& Thoms. }\end{array}$ & Gulancha & Menispermaceae & Clim & $\mathrm{R}$ & Jan-Oct & AA 43 \\
\hline Acacia catcechu (L.f.) Willd. & Khair & Mimosaceae & Tree & $\mathrm{R}$ & Mar-Dec & AA 12 \\
\hline Acacia nilotica (L.) Del. & Babla & Mimosaceae & Tree & $\mathrm{C}$ & Apr-Aug & AA 145 \\
\hline Albizia procera (Roxb.) Benth. & Silkoroi & Mimosaceae & Tree & $\mathrm{C}$ & Jan-Dec & AA 53 \\
\hline Mollugo pentahylla $\mathrm{L}$. & $\begin{array}{l}\text { Khetpapr } \\
\text { a }\end{array}$ & Molluginaceae & Herb & $\mathrm{C}$ & Jun-Jan & AA 85 \\
\hline $\begin{array}{l}\text { Artocurpus lacucha Buch- } \\
\text { Ham. }\end{array}$ & Dewa & Moraceae & Tree & $\mathrm{T}$ & Apr-Jun & AA 146 \\
\hline $\begin{array}{ll}\text { Artocurpus heterophyllus } \\
\text { Lamk. }\end{array}$ & Kathal & Moraceae & Tree & $\mathrm{C}$ & Feb-Jul & AA 94 \\
\hline Ficus hispida L.f. & Khoksa & Moraceae & Tree & $\mathrm{C}$ & Apr-Sep & AA 57 \\
\hline Ficus racemosa $\mathrm{L}$. & \begin{tabular}{|l|} 
Jagdumur \\
\end{tabular} & Moraceae & Tree & $\mathrm{C}$ & Sep-Nov & AA 13 \\
\hline Ficus religiosa $\mathrm{L}$. & Pakur & Moraceae & Tree & $\mathrm{C}$ & Mar-Oct & AA 27 \\
\hline Ficus benghalensis L. & Bot & Moraceae & Tree & $\mathrm{C}$ & May-Aug & AA 61 \\
\hline Sterblus asper Lour. & Sheora & Moraceae & Tree & $\mathrm{R}$ & Feb-Jun & AA 100 \\
\hline Moringa oleifera Lamk. & Sajna & Moringaceae & Tree & $\mathrm{C}$ & Jan-Dec & AA 66 \\
\hline Musa paradisiaca $\mathrm{L}$. & Kola & Musaceae & Herb & $\mathrm{C}$ & Jan-Dec & AA 101 \\
\hline Psidium guajava $\mathrm{L}$. & Peyara & Myrtaceae & Tree & $\mathrm{C}$ & Jan-Dec & AA 70 \\
\hline $\begin{array}{l}\text { S. samarangense (Blume) } \\
\text { Merr \& Perry }\end{array}$ & Jamrul & Myrtaceae & Tree & $\mathrm{C}$ & Feb-Mar & AA 34 \\
\hline Syzygium cumini (L.) Skeels. & Jam & Myrtaceae & Tree & $\mathrm{C}$ & Mar-Jun & AA 173 \\
\hline Averrhoa carambola $\mathrm{L}$. & \begin{tabular}{|l|} 
Kamrang \\
a \\
\end{tabular} & Oxalidaceae & Tree & $\mathrm{C}$ & Sep-Mar & AA 72 \\
\hline Oxalis corniculata $\mathrm{L}$. & Amrul & Oxalidaceae & Herb & $\mathrm{C}$ & Sep-May & AA 24 \\
\hline Orobanche agyptica Pers. & $\begin{array}{l}\text { Orobanch } \\
\mathrm{i}\end{array}$ & Orobanchaceae & Herb & $\mathrm{C}$ & Nov-Mar & AA 210 \\
\hline Argemone mexicana $\mathrm{L}$. & $\begin{array}{l}\text { Sheyal } \\
\text { kata }\end{array}$ & Papaveraceae & Herb & $\mathrm{C}$ & Feb-Jun & AA 01 \\
\hline $\begin{array}{l}\text { Peperomia pellucida (L.) H.B } \\
\& \text { K. }\end{array}$ & Iuchi Pata & Piperaceae & Herb & $\mathrm{C}$ & Jul-Sep & AA 149 \\
\hline Bambusa bambos (L.) Voss. & Bash & Poaceae & Shrub & $\mathrm{C}$ & Jan-Dec & AA 74 \\
\hline Cynodon dactylon (L.) Pers. & Durba & Poaceae & Herb & $\mathrm{C}$ & Jan-Dec & AA 67 \\
\hline Zea mays $\mathrm{L}$. & Vutta & Poaceae & Shrub & $\mathrm{C}$ & Mar-Apr & AA 163 \\
\hline $\begin{array}{l}\text { Persicaria hydropiper (L.) } \\
\text { Spach. }\end{array}$ & Biskatali & Polygonaceae & Herb & $\mathrm{C}$ & Aug-Apr & AA 03 \\
\hline Portulaca oleracea L. & \begin{tabular}{|l|} 
Baranuni \\
a \\
\end{tabular} & Portulacaceae & Herb & $\mathrm{C}$ & May-Jul & AA 28 \\
\hline Punica granatum $\mathrm{L}$. & Dalim & Punicaceae & Shrub & $\mathrm{C}$ & Jan-Dec & AA 62 \\
\hline Zizyphus mauritiana Lamk. & Boroi & Rhamnaceae & Tree & $\mathrm{C}$ & Sep-Jan & AA 128 \\
\hline Rosa centifolia $\mathrm{L}$. & Golap & Rosaceae & Shrub & $\mathrm{C}$ & Jan-Dec & AA 164 \\
\hline Ixora coccinea $\mathrm{L}$. & Rongon & Rubiaceae & Shrub & $\mathrm{C}$ & Jan-Dec & AA 39 \\
\hline $\begin{array}{ll}\text { Neolamarckia } & \text { cadamba } \\
\text { (Roxb.) Bosser. } & \\
\end{array}$ & Kadom & Rubiaceae & Tree & $\mathrm{C}$ & May-Jul & AA 63 \\
\hline Aegle marmelos (L.) Corr. & Bel & Rutaceae & Tree & C & Apr-Dec & AA 90 \\
\hline $\begin{array}{l}\text { Citrus aurantifolia (Christm. } \\
\text { \& Panzer) Swingle. }\end{array}$ & Labu & Rutaceae & Shrub & C & Mar-Sep & AA 122 \\
\hline Citrus maxima (Burm.) Merr. & $\begin{array}{l}\text { Jambura } \\
\end{array}$ & Rutaceae & Tree & $\mathrm{C}$ & Feb-Nov & AA 89 \\
\hline
\end{tabular}

Copyright (C 2020-2021, Journal of Scientific Research in Medical and Biological Sciences (JSRMBS), Under the license CC BY- 4.0 


\begin{tabular}{|c|c|c|c|c|c|c|}
\hline Limonia acidissima $\mathrm{L}$. & Kothbel & Rutaceae & Tree & $\mathrm{C}$ & Feb-Dec & AA 106 \\
\hline Murraya paniculata (L.) Jack. & Kamini & Rutaceae & Shrub & $\mathrm{C}$ & Mar-Jan & AA 138 \\
\hline Litchi chinensis Sonn. & Lichu & Sapindaceae & Tree & $\mathrm{C}$ & Apr-Jun & AA 102 \\
\hline $\begin{array}{l}\text { Manikara zapota (L.) P. van } \\
\text { Roven. }\end{array}$ & Sofeda & Sapotaceae & Tree & $\mathrm{C}$ & May-Jun & AA 45 \\
\hline Mimusops elengi $\mathrm{L}$. & Bokul & Sapotaceae & Tree & $\mathrm{C}$ & Mar-Jun & AA 64 \\
\hline Scoparia dulcis L. & Bondone & Scrophulariaceae & Herb & $\mathrm{C}$ & Jan-Dec & AA 21 \\
\hline Smilax macrophylla Roxb. & $\begin{array}{l}\text { Kumari } \\
\text { lata }\end{array}$ & Smilaceae & Clim & $\mathrm{R}$ & Nov-Mar & AA 49 \\
\hline Capsicum frutescens $\mathrm{L}$. & Morich & Solanaceae & Herb & $\mathrm{C}$ & Jan-Dec & AA 46 \\
\hline Datura metel L. & Dhatura & Solanaceae & Shrub & $\mathrm{C}$ & Jan-Dec & AA 104 \\
\hline Lycopersicon esculentum Mill. & Tomato & Solanaceae & Herb & $\mathrm{C}$ & Sep-Apr & AA 118 \\
\hline $\begin{array}{l}\text { Nicotiana plumbaginifolia } \\
\text { Viv. }\end{array}$ & $\begin{array}{l}\text { Bontama } \\
\mathrm{k}\end{array}$ & Solanaceae & Herb & $\mathrm{C}$ & Jan-Dec & AA 127 \\
\hline Physalis minima $\mathrm{L}$. & $\begin{array}{l}\text { Kopal } \\
\text { Phutki }\end{array}$ & Solanaceae & Herb & $\mathrm{C}$ & Jan-Dec & AA 02 \\
\hline Solanum nigrum $\mathrm{L}$. & Titbegun & Solanaceae & Herb & $\mathrm{C}$ & Jan-Dec & AA 20 \\
\hline Solanum torvum Swartz. & $\begin{array}{l}\text { Gota } \\
\text { Begun }\end{array}$ & Solanaceae & Shrub & $\mathrm{C}$ & Dec-Feb & AA 16 \\
\hline Solanum tuberosum $\mathrm{L}$. & Golalu & Solanaceae & Herb & $\mathrm{C}$ & Oct-Feb & AA 08 \\
\hline Solanum virginianum $\mathrm{L}$. & Kantakari & Solanaceae & Herb & $\mathrm{C}$ & Oct-Feb & AA 51 \\
\hline Solanum melongena $\mathrm{L}$. & Begun & Solanaceae & Herb & $\mathrm{C}$ & Oct-Feb & AA 04 \\
\hline Abroma augusta (L.) L.f. & $\begin{array}{l}\text { Ulat } \\
\text { kambal }\end{array}$ & Sterculiaceae & Shrub & $\mathrm{T}$ & Jun-Dec & AA 80 \\
\hline Trema orientalis (L.) Blume. & Jibon & Ulmaceae & Tree & $\mathrm{C}$ & Jan-Jun & AA 06 \\
\hline Pouzolzia zeylanica (L.) Benn. & kullaruki & Urticaceae & Herb & $\mathrm{C}$ & Jan-Dec & AA 81 \\
\hline $\begin{array}{l}\text { Clerodendrum inerme (L.) } \\
\text { Grentn. }\end{array}$ & $\begin{array}{l}\text { Bamun } \\
\text { hati }\end{array}$ & Verbenaceae & Shrub & $\mathrm{C}$ & Jul-Nov & AA 10 \\
\hline Clerodendrum viscosum Vent. & Bhat & Verbenaceae & Shrub & $\mathrm{C}$ & Jan-Jul & AA 107 \\
\hline Duranta repens $\mathrm{L}$. & $\begin{array}{l}\text { Kiilo } \\
\text { mehedi }\end{array}$ & Verbenaceae & Shrub & $\mathrm{C}$ & Jan-Dec & AA 11 \\
\hline Lantana camara $\mathrm{L}$. & Chotra & Verbenaceae & Shrub & $\mathrm{C}$ & Jan Dec & AA 22 \\
\hline Phyla nodiflora (L.) Greene & $\begin{array}{l}\text { Khudi } \\
\text { okra }\end{array}$ & Verbenaceae & Herb & $\mathrm{C}$ & Jan-Dec & AA 83 \\
\hline Vitex negunda L. & Nisinda & Verbenaceae & Shrub & $\mathrm{R}$ & Apr-Feb & AA 86 \\
\hline Vitis trifolia (L.) Domin. & Amallata & Vitaceae & Clim & $\mathrm{R}$ & Jan-Dec & AA 69 \\
\hline Cucurma longa $\mathrm{L}$. & Holud & Zingiberaceae & Herb & $\mathrm{C}$ & Mar-Oct & AA 132 \\
\hline Zingiber officinale Roscoe & Ada & Zingiberaceae & Herb & $\mathrm{C}$ & Mar-Aug & AA 125 \\
\hline
\end{tabular}

Jan = January, Feb = February, Mar = March, Apr = April, Ma = May, Jun = June,$J u l=J u l y$, Aug = August, Sep $=$ September, Oct $=$ October, Nov $=$ November, Dec $=$ December,$\quad$ Clim $=$ Climber,$\quad C=$ Common, $T=$ Threatened species, $R=$ Rare

\section{No.of. species}

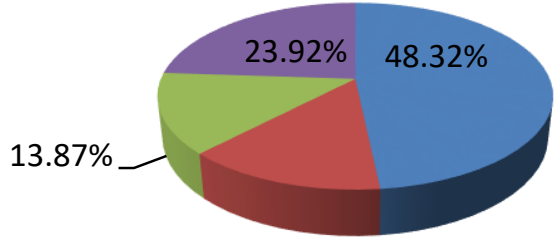

- Herb

Shrub

Climber

$13.87 \%$

Figure 1: Habit diversity of the recorded species 


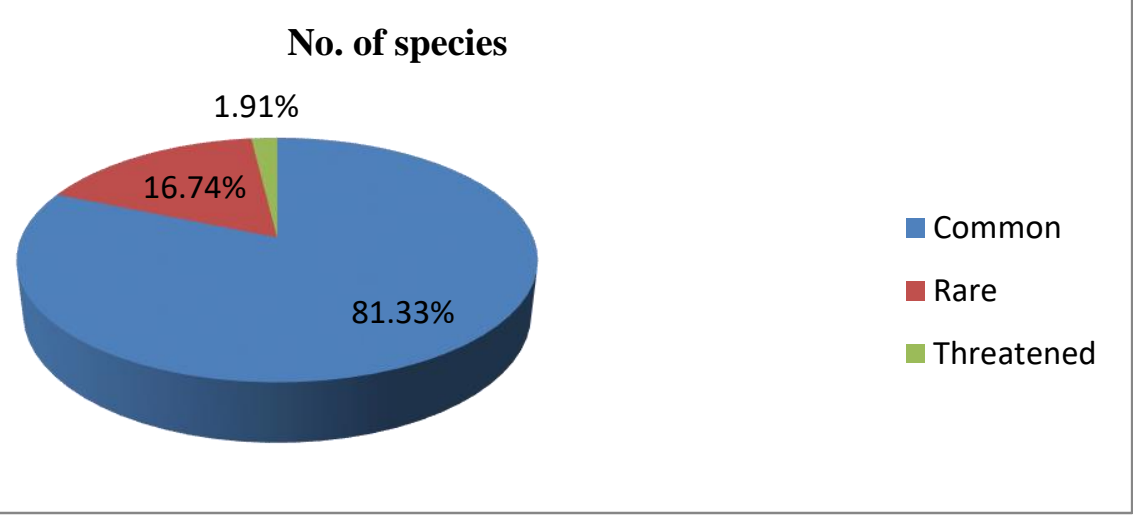

Figure 2: Recorded status of occurrence in the study area

Table 3: Medicinal plants used by the local people in Chaar Khidirpur area of Rajshahi.

\begin{tabular}{|c|c|c|c|c|c|}
\hline Scientific name & $\begin{array}{l}\text { Local } \\
\text { name }\end{array}$ & Family name & Habit & $\begin{array}{l}\text { Parts } \\
\text { used }\end{array}$ & Medicinal Uses \\
\hline $\begin{array}{l}\text { Aloe indica (L.) } \\
\text { Burm. f. }\end{array}$ & $\begin{array}{l}\text { Ghrita } \\
\text { kumari }\end{array}$ & Aloceae & Herb & Leaf & $\begin{array}{l}\text { The juice of leaves is used for } \\
\text { hair disease, constipation, } \\
\text { weakness, sexual disease and } \\
\text { burning sensation. }\end{array}$ \\
\hline $\begin{array}{l}\text { Spondius pinnata } \\
\text { (L.f) Kurz }\end{array}$ & Aamra & Anacardiaceae & Tree & $\begin{array}{l}\text { Bark, } \\
\text { fruit }\end{array}$ & $\begin{array}{l}\text { The bark is used in the } \\
\text { treatment of stomach aches } \\
\text { and dysentery. Fruit is used } \\
\text { as fever. }\end{array}$ \\
\hline $\begin{array}{l}\text { Annona squamosa } \\
\text { L. }\end{array}$ & Aafa & Annonaceae & Tree & Fruit & $\begin{array}{l}\text { Fruit is used as astringents in } \\
\text { diarrhea and dysentery. }\end{array}$ \\
\hline $\begin{array}{l}\text { Polyalthia } \\
\text { longifolia Benth } \\
\text { Hook }\end{array}$ & Debdarue & Annonaceae & Tree & $\begin{array}{l}\text { Stem, } \\
\text { bark, } \\
\text { leaf }\end{array}$ & $\begin{array}{l}\begin{array}{l}\text { Stem, bark, leaves are } \\
\text { exhibits } \\
\text { properties. }\end{array} \\
\text { antioxidant }\end{array}$ \\
\hline $\begin{array}{l}\text { Centella asiatica } \\
\text { (L.) Urb }\end{array}$ & Thankuni & Apiceae & Herb & Leaf & $\begin{array}{l}\text { Leaf is used is alternative, } \\
\text { astringent, tonic and diuretic. }\end{array}$ \\
\hline $\begin{array}{l}\text { Carissa carandas } \\
\text { (L.) K. schum }\end{array}$ & Karomcha & Apocynaceae & Herb & Fruit & $\begin{array}{l}\text { The fruit has been used } \\
\text { remedy for diabetes. }\end{array}$ \\
\hline $\begin{array}{l}\text { Catharanthus } \\
\text { roseus } \mathrm{L} .\end{array}$ & Noyontara & Apocynaceae & Herb & Leaf & $\begin{array}{l}\text { Leaf juice is applied to wasp- } \\
\text { sting. }\end{array}$ \\
\hline $\begin{array}{ll}\text { Rauvolfia } & \\
\text { serpentina } & \text { (L.) } \\
\text { Benth } & \end{array}$ & Sarpa gandha & Apocynaceae & Shrub & Root & $\begin{array}{l}\text { Root juice is a valuable } \\
\text { remedy in high blood } \\
\text { pressure. }\end{array}$ \\
\hline $\begin{array}{l}\text { Calotropis } \\
\text { gigantean L. }\end{array}$ & Akondo & Asclepiadaceae & Shrub & Root & $\begin{array}{l}\text { Root bark is useful for } \\
\text { constipation and indigestion. }\end{array}$ \\
\hline Tagetes patula $\mathrm{L}$. & $\begin{array}{l}\text { Genda } \\
\text { phul }\end{array}$ & Asteraceae & Herb & Leaf & $\begin{array}{l}\text { Leaves are good for kidney } \\
\text { muscular pair and piles. }\end{array}$ \\
\hline Carica papaya $L$. & pepe & Caricaceae & Tree & Root & $\begin{array}{l}\text { Root extract is used for } \\
\text { cancer of the uterus and } \\
\text { syphilis. }\end{array}$ \\
\hline Terminalia arjuna & Arjun & Combretaceae & Tree & Stem & $\begin{array}{l}\text { Stem juice is used for } \\
\text { prevention of heart disease. }\end{array}$ \\
\hline $\begin{array}{l}\text { Commelina } \\
\text { benghalensis }\end{array}$ & Kanshira & Commelinaceae & Herb & Leaf & $\begin{array}{l}\text { Leaf juice is used to prevent } \\
\text { urinary problem. }\end{array}$ \\
\hline $\begin{array}{l}\text { Benincasa hispida } \\
\text { (Thurb) cogn. }\end{array}$ & $\begin{array}{l}\text { Chal } \\
\text { kumra }\end{array}$ & Cucurbitaceae & climber & Fruit & $\begin{array}{l}\text { Fruits are laxative and } \\
\text { diuretic. }\end{array}$ \\
\hline
\end{tabular}




\begin{tabular}{|c|c|c|c|c|c|}
\hline Cucumis melo L. & Bhangi & Cucurbitaceae & climber & Fruit & $\begin{array}{l}\text { The pulp of the fruit is used } \\
\text { as diuretic. }\end{array}$ \\
\hline $\begin{array}{l}\text { Cucurbita maxima } \\
\text { Duch }\end{array}$ & $\begin{array}{l}\text { Misti } \\
\text { kumra }\end{array}$ & Cucurbitaceae & climber & Fruit & $\begin{array}{l}\text { The fruit pulp is used as a } \\
\text { poultice to burns. }\end{array}$ \\
\hline $\begin{array}{l}\text { Luffa acutangula } \\
\text { (L.) Roxb }\end{array}$ & Jhinga & Cucurbitaceae & climber & Leaf & $\begin{array}{l}\text { Leaf paste is applied to } \\
\text { leprosy haemorrhoides. }\end{array}$ \\
\hline $\begin{array}{l}\text { Luffa cylindrica } \\
\text { (L.) Roem }\end{array}$ & koror & Cucurbitaceae & climber & Fruit & $\begin{array}{l}\text { Fruits are expectorant tonic, } \\
\text { laxative and diuretic. }\end{array}$ \\
\hline $\begin{array}{l}\text { Trichossasthes } \\
\text { dioica } \text { Roxb }\end{array}$ & Potol & Cucurbitaceae & climber & Leaf & The leaves are antepyratic. \\
\hline $\begin{array}{l}\text { Elaeocarpus } \\
\text { robustus Roxb }\end{array}$ & Jolpai & Elaeocarpaceae & Tree & Fruit & $\begin{array}{l}\text { Fruit is used in dysentery, } \\
\text { diabetes and diarrhea. }\end{array}$ \\
\hline Acalypha indica $\mathrm{L}$. & $\begin{array}{l}\text { Muktajhur } \\
\mathrm{i}\end{array}$ & Euphrbiaceae & Herb & $\begin{array}{l}\text { Whole } \\
\text { plant }\end{array}$ & $\begin{array}{l}\text { Whole plant paste is used } \\
\text { skin diseases, eczema, and } \\
\text { skin fungal infection. }\end{array}$ \\
\hline $\begin{array}{l}\text { Croton } \\
\text { bonplandianus Bail }\end{array}$ & Banmorich & Euphrbiaceae & Herb & Seed & $\begin{array}{l}\text { Seed paste is applied locally } \\
\text { on eczema and ringworm. }\end{array}$ \\
\hline Euphorbia hirta L. & Dudhiya & Euphorbiaceae & Herb & $\begin{array}{l}\text { Whole } \\
\text { plant }\end{array}$ & $\begin{array}{l}\text { The plant is astringent and } \\
\text { haemostatic. }\end{array}$ \\
\hline $\begin{array}{l}\text { Sesamum indicum } \\
\mathrm{L} .\end{array}$ & Tili & Pedaliaceae & Herb & Seed & Seeds are used against piles. \\
\hline $\begin{array}{l}\text { Leucas aspera } \\
\text { (wild) Link. }\end{array}$ & Shetodron & Lamiaceae & Herb & Leaf & $\begin{array}{l}\text { Juice of leaves is applied in } \\
\text { skin eruption. }\end{array}$ \\
\hline $\begin{array}{l}\text { Ocimum } \\
\text { tenuiflorum } \mathrm{L} .\end{array}$ & Tulsi & Lamiaceae & Shrub & Leaf & $\begin{array}{l}\text { Leaf juice is used in cough } \\
\text { and cold. }\end{array}$ \\
\hline $\begin{array}{l}\text { Lawsonia inermis } \\
\text { Linn }\end{array}$ & Mehedi & Lythraceae & Shrub & Leaf & $\begin{array}{l}\text { Leaf paste is used in skin } \\
\text { diseases. }\end{array}$ \\
\hline $\begin{array}{l}\text { Abelmoschus } \\
\text { esculentus }(\mathrm{L} .) \\
\text { moench }\end{array}$ & Bhindi & Malvaceae & Herb & Fruit & $\begin{array}{l}\text { Fruits are cooling stomachic, } \\
\text { astringent and diarrhea. }\end{array}$ \\
\hline $\begin{array}{l}\text { Acacia cathechu } \\
\text { (L.f.) wild }\end{array}$ & Khair & Mimosaceae & Tree & Bark & $\begin{array}{l}\text { Bark is astringent, } \\
\text { antidysenteric. }\end{array}$ \\
\hline Ficus hispida L.f. & khoksa & Moraceae & Tree & Leaf & It is useful in piles, anemia. \\
\hline Psidium guajava $\mathrm{L}$. & Peyara & Myrtaceae & Tree & Root & $\begin{array}{l}\text { Root paste mixed with water } \\
\text { used to treat diarrhea and } \\
\text { dysentery. }\end{array}$ \\
\hline $\begin{array}{l}\text { Syzygium } \\
\text { cumini(L.) skeels }\end{array}$ & Jam & Myrtaceae & Tree & Fruit & $\begin{array}{l}\text { it is good for bronchitis, } \\
\text { asthma \& dysentery. }\end{array}$ \\
\hline Punica granatum L. & Dalim & Punicaceae & Shrub & Stem & $\begin{array}{l}\text { Young stem is used for } \\
\text { abdominal pain. }\end{array}$ \\
\hline $\begin{array}{l}\text { Aegle marmelos } \\
\text { (L.) corr }\end{array}$ & Bel & Rutaceae & Tree & Fruit & $\begin{array}{l}\text { Unripe fruit is used in } \\
\text { diarrhea, dysentery and ripe } \\
\text { fruit is used for constipation. }\end{array}$ \\
\hline $\begin{array}{l}\text { Citrus aurantifolia } \\
\text { L. }\end{array}$ & Labu & Rutaceae & Tree & Fruit & $\begin{array}{l}\text { Fruits are used against skin } \\
\text { irritation and nausea. }\end{array}$ \\
\hline $\begin{array}{l}\text { Citrus maxima } \\
\text { (Burm.) Merr. }\end{array}$ & Jambura & Rutaceae & Tree & Fruit & $\begin{array}{l}\text { Fruit is useful in vomiting } \\
\text { and diarrhea. }\end{array}$ \\
\hline $\begin{array}{l}\text { Limonia acidissima } \\
\text { L. }\end{array}$ & Kodbel & Rutaceae & Tree & Fruit & $\begin{array}{l}\text { Fruit is stimulant, astringent, } \\
\text { aphrodisiac, diuretic, cardio- } \\
\text { tonic to the livers and lungs. }\end{array}$ \\
\hline $\begin{array}{ll}\text { Litchi } & \text { chinensis } \\
\text { Sonn } & \\
\end{array}$ & Lichu & Sapindaceae & Tree & Fruit & $\begin{array}{l}\text { Fruits are tonic to the heart, } \\
\text { brain and liver. }\end{array}$ \\
\hline $\begin{array}{l}\text { Capsicum } \\
\text { frutescens } \mathrm{L} .\end{array}$ & morich & Solanaceae & Herb & Leaf & $\begin{array}{l}\text { Leaves are used in headache, } \\
\text { pain, night blindness. }\end{array}$ \\
\hline
\end{tabular}




\begin{tabular}{|l|l|l|l|l|l|}
\hline $\begin{array}{l}\text { Nicotiana } \\
\text { plumbaginifolia } \text { viv }\end{array}$ & Bontamak & Solanaceae & Herb & Leaf & $\begin{array}{l}\text { Leaf juice is used for skin } \\
\text { disease. }\end{array}$ \\
\hline Physalis minima L. & kopaittka & Solanaceae & Herb & Fruit & $\begin{array}{l}\text { Fruits are tonic, diuretic and } \\
\text { purgative. }\end{array}$ \\
\hline $\begin{array}{l}\text { Solanum } \\
\text { melongena } \text { L. }\end{array}$ & Begum & Solanaceae & shrub & Fruit & $\begin{array}{l}\text { Unripe fruit is laxative, } \\
\text { analgesic, cardio-tonic. }\end{array}$ \\
\hline Abroma augusta L. & Ulatkambal & Sterculiaceae & shrub & Root & $\begin{array}{l}\text { Root bark is used in irregular } \\
\text { menses pain. }\end{array}$ \\
\hline Vitex negundo L. & Nisinda & Verbenaceae & Shrub & Leaf & $\begin{array}{l}\text { Leaves are tonic, } \\
\text { vermifuge \& anti-parasite. }\end{array}$ \\
\hline $\begin{array}{l}\text { Zingiber officinale } \\
\text { Rose. }\end{array}$ & Ada & Zingiberaceae & Herb & $\begin{array}{l}\text { Rhizo } \\
\text { me }\end{array}$ & $\begin{array}{l}\text { Rhizome juice It's used in } \\
\text { constipation, dysentery and } \\
\text { vomiting. }\end{array}$ \\
\hline
\end{tabular}

\section{Conclusion and Suggestion}

The diversity of angiosperm taxa in the Chaar Khidirpur area of Rajshahi, Bangladesh was recorded. The total numbers of the recorded species of angiosperms were 177 genera belonging to 71 families. The distribution of angiosperm plant species in the families shows variation. Asteraceae was represented by 19 species. Cucurbitaceae was represented by 17 species. Fabaceae and Euphorbiaceae were represented by 11 species each. Solanaceae was represented by 10 species. Acanthaceae was represented by 9 species. A single species was represented by 32 families while 2 to 7 species was represented by 33 families. The following species were found rarely distributed in the study area like Vitex negunda L., Stephania japonica (Thunb.) Miers., Exacum pedunculatum L., Dillenia indica L., Senna alata (L.) Roxb., Justicia adhatoda L., Hygrophila schulli (Buch-Ham.) M.R. \& S.N.Almeida., Thunbergia grandiflora (Roxh. ex Rotller) Roth., Alstonia scholaris (L.) R. Br., Rauvolfia serpentina (L.) Benth ex Kurz., Chromolaena odorata L. King. \& Robinson., Eclipta alba (L.) Hassk., Grangea maderaspatana (L.) Poir., Spilanthes acmella (L.)L., Synedrella nodiflora (L.) Gaertn., Youngia japonica (L.) DC., Cleome viscosa L., Costus speciosus (Koenig ex Retz) Smith., Kalanchoe laciniala (L.) Pers., Gymnopetalum cochinchinense (Lour.) Kurz., Mukia maderaspatana (L.) M. Roem., Trichossasthes tricuspidata Lour., Dioscorea alata L., Elaeocarpus tectorius (Lour.) Poir., Jatropha curcas L., Phyllanthus emblica L., Melilotus alba Desr., Exacum pedunculatum L., Ocimum tenuiflorum L., Asparagus racemosus Willd., Stephania japonica (Thunb.) Miers., Tinospora cordifolia (Willd.) Hook. f \& Thoms. and Dirgeria muricata (L.) Mart., Abroma augusta (L.) L.f., Artocurpus lacucha Buch-Ham., Litsea glutinosa (Lour.) Rob. are threatened species in the study area. Forty-five (45) medicinal plants were used for the treatment of more than 61 diseases. These medicinal plants were useful in the discovery of new herbal medicines. All collected specimens were studied, identified and have been lodged in the Herbarium, Department of Botany, University of Rajshahi, Bangladesh.

\section{Acknowledgements}

The authors are grateful to the local people in Chaar Khidirpur area of Rajshahi, Bangladesh for their co-operation and help during the research work. 


\section{Conflict of Interest}

None declared.

\section{Funding:}

This study has not received any external funding.

\section{Data and Materials Availability}

All data associated with this study are presented in this paper.

\section{Peer-review}

External peer-review was done through double blind method.

\section{References}

Ahmed, Z.U., Begum, Z.N.T., Hassan, M.A., Khondker, M., Kabir, S.M.H., Ahmad, M., Ahmed, A.T.A., Rahman, A.K.A. and Haque, E.U.(Eds). (2008-2009). Encyclopedia of Flora and Fauna of Bangladesh. Vols. 6-10. Angiosperms Asiatic Society of Bangladesh, Dhaka.

Anisuzzaman, M., Rahman, A.H.M.M., Rashid, M.H., Naderuzzaman, A.T.M. and Islam A.K.M.R. (2007). An Ethnobotanical Study of Madhupur, Tangail. Journal of Applied Sciences Research, 3(7): 519-530.

Arefin, M.K., M.M. Rahman, M.Z. Uddin and M.A. Hassan (2011). Angiosperm Flora of Satchari National Park, Habiganj, Bangladesh.Bangladesh J. Plant Taxon., 18(2): 117140.

Bangladesh Population Census (BPC). (2001). Bangladesh Bureau of Statistics; Rajshahi City Corporation 2007.

Choudhury, A. R. and Rahmatullah, M. (2012). Ethnobotanical study of wound healing plants among the folk medicinal practioners several district in Bangladesh. AmericanEurasian Journal of Sustainable Agriculture, 6(4), 371-377.

Faruque, M. O. and Uddin, S.B. (2014). Ethnomedicinal study of the Marma community of Bandarban district of Bangladesh. Academia Journal of Medicinal Plants, 2(2), $014-$ 025.

Ghani, A. (2003). Medicinal Plants of Bangladesh. Asiatic Society of Bangladesh, Dhaka.

Hooker, J. D. (1877). (rep. ed. 1961). Flora of British India. Vols.1-7. L. Reeve and Co. Ltd. London, U.K.

Huq, A.M. (1986). Plant Names of Bangladesh. Bangladesh National Herbarium, BARC, Dhaka, Bangladesh. 
Islam, M.R., Uddin,M.Z. and Hassan, M.A. (2009). An Assessment of the Angiosperm Flora of Ramgarh Upazilla of Khagrachhari District, Bangladesh. Bangladesh J. Plant Taxon. 16(2): 115-140.

Kirtikar, K.R. and Basu, B.D. (1987). Indian Medicinal Plants. Vols. 1-4. Lalit Mohan Basu, Allahabad, Jayyed Press, New Delhi, India.

Kona, S. and Rahman, A.H.M.M. (2015). An Assessment of Angiosperm Diversity at Mahadebpur Upazila of Naogaon District, Bangladesh. International Journal of Advanced Research. India. 3(10): 1067-1086.

Pasha, M.K. and S.B.Uddin. (2013). Dictionary of Plant Names of Bangladesh (Vascular Plants). Janokalyan Prokashani. Chittagong, Dhaka, Bangladesh.

Prain, D. (1903) (rep. ed. 1963). Bengal Plants. Vols.1-2. Botanical Survey of India. Calcutta, India.

Rahman, A.H.M.M. (2017). Annotated List in the Graveyards Trees of Rajshahi City, Bangladesh. Discovery. 53(254): 107-116.

Rahman, A.H.M.M. (2013). Angiospermic flora of Rajshahi district, Bangladesh. American Journal of Life Sciences, 1(3): 105-112.

Rahman, A.H.M.M. (2021). Folk Medicinal Plants Used by Local Herbalists in and around Rajshahi Metropolitan City, Bangladesh, Journal of Botanical Research, 3(2):20-30.

Rahman, A.H.M.M. (2021b). Asteraceae: A Taxonomically and Medicinally Important Sunflower Family. American International Journal of Biology and Life Sciences, 3(1):1-17.

Rahman, A.H.M.M. and Keya, M.A. (2014). Assessment of Angiosperm Flora at the Village Sabgram under Sadar Upazila of Bogra District, Bangladesh. International Journal of Advanced Research. India. 2(11): 443-458.

Rahman, A.H.M.M. and Khatun, M.M. (2020). Leafy Vegetables in Chapai Nawabganj District of Bangladesh Focusing on Medicinal Value. Bangladesh Journal of Plant Taxonomy 27(2):359-375.

Rahman, A.H.M.M., Alam, M.S., Hossain, M.B., Nesa, M.N., Islam, A.K.M.R. and Rahman, M.M. (2008). Study of Species Diversity on the family Asteraceae (Compositae) of the Rajshahi Division. Research Journal of Agriculture and Biological Sciences. Pakistan. 4(6): 794-797.

Rahman, A.H.M.M., Anisuzzaman, M., Ahmed, F., Zaman, A.T.M.N. and Islam, A.K.M. R. (2007). A Floristic Study in the Graveyards of Rajshahi City. Research Journal of Agriculture and Biological Sciences. Pakistan. 3(6): 670-675.

Rahman, A.H.M.M., Ferdous, Z. and Islam, A. K. M. R. (2014). A Preliminary Assessment of Angiosperm Flora of Bangladesh Police Academy. Research in Plant Sciences. 2(1): 9-15.

Rahman, A.H.M.M., Ferdows, Z, Nitu, S.K. and Islam, A.K.M.R. (2015). Herbaceous Plant Species in and around Rajshahi Metropolitan City, Bangladesh. International Journal of Advanced Research. India. 3(5): 1002-1018.

Sarker, A.K. and Rahman, A.H.M.M. (2016). A Preliminary Checklist of Angiosperm Flora at Katakhali Pouroshova of Rajshahi, Bangladesh. Discovery. 52(251): 2127-2140.

Sarker, P and Rahman, A.H.M.M. (2019). Angiosperms in Gobindaganj Upazila of Gaibandha

District, Bangladesh. Bangladesh Journal of Plant Taxonomy, 26(2): 285-298 
Sultana, R. and Rahman, A.H.M.M. (2016). Convolvulaceae: A Taxonomically and Medicinally Important Morning Glory Family. International Journal of Botany Studies. 1(3): 47-52.

Tutul, E., Uddin, M.Z., Rahman, M.O. and Hassan, M.A. (2010). Angiospermic Flora of Runctia Sal Forest, Bangladesh. II. Magnoliopsida (Dicots). Bangladesh J. Plant Taxon. 17(1): 33-54.

Uddin, M., Roy, S., Hassan, M. A. and Rahman, M.M. (2008). Medicobotanical report on the Chakma people of Bangladesh. Bangladesh Journal of Plant Taxonomy, 15(1), 6772.

Uddin, M.Z. and Hassan, M.A. (2010). Angiosperm Diversity of Lawachara National Park (Bangladesh): A Preliminary Assessment. Bangladesh J. Plant Taxon. 17(1): 9-22.

Yusuf, M., Begum, J., Hoque, M. N. and Choudhury, J. U. (2009). Medicinal plants of Bangladesh-Revised and Enlarged. Bangladesh Coun. Sci. Ind. Res. Lab. Chittagong, Bangladesh. 